\title{
IL-27 controls sepsis-induced impairment of lung antibacterial host defence
}

\author{
Ju Cao, ${ }^{1}$ Fang $\mathrm{Xu}_{1}{ }^{2}$ Shihui Lin, ${ }^{2}$ Zhixin Song, ${ }^{3}$ Lipin Zhang, ${ }^{1}$ Peng Luo, ${ }^{1}$ \\ Huajian $\mathrm{Xu}_{1}{ }^{1}$ Dairong Li, ${ }^{4} \mathrm{Ke}$ Zheng, ${ }^{5}$ Guosheng Ren, ${ }^{5}$ Yibing Yin ${ }^{3}$
}

\begin{abstract}
- Additional material is published online only. To view please visit the journal online (http://dx.doi.org/10.1136/ thoraxjnl-2014-205777)

${ }^{1}$ Department of Laboratory Medicine, The Affiliated Hospital of Chongqing Medical University, Chongaing Medical University, Chongqing, China ${ }^{2}$ Department of Emergency and Intensive Care Unit, The Affiliated Hospital of Chongqing Medical University, Chongqing Medical University, Chongqing, China

${ }^{3}$ Key Laboratory of Diagnostic Medicine designated by the Ministry of Education, Chongqing Medical University, Chongqing, China

${ }^{4}$ Department of Respiratory Disease, The First Affiliated Hospital of Chongqing Medical University, Chongqing, China ${ }^{5}$ Molecular Oncology and Epigenetics Laboratory, The First Affiliated Hospital of Chongqing Medical University, Chongqing, China
\end{abstract}

Correspondence to Dr Ju Cao, Department of Laboratory Medicine,

The Affiliated Hospital of Chongqing Medical University, Chongqing Medical University, No. 1 Youyi Road, Yuzhong District, Chongqing 400016, China;

caoju723@163.com

$\mathrm{JC}$ and FX are contributed equally.

Received 20 May 2014 Accepted 27 June 2014 Published Online First 29 July 2014

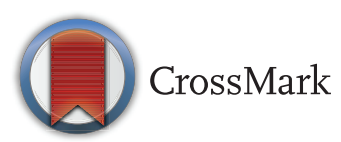

To cite: Cao J, Xu F, Lin S, et al. Thorax 2014;69: 926-937.

\section{ABSTRACT}

Background Interleukin 27 (IL-27) is an important cytokine regulating host immune responses. However, its role in sepsis-induced immunosuppression remains unclear.

Aim To investigate the role of IL-27 in modulating sepsis-induced immunosuppression using a murine model of caecal ligation and puncture (CLP)-induced sepsis followed by secondary challenge with

Pseudomonas aeruginosa.

Methods CLP or sham surgery was performed in wild-type (WT) and IL-27 receptor (IL-27R)/WSX-1 knockout (KO) mice, and then mice were infected with intratracheal $P$ aeruginosa.

Results IL-27 was upregulated in patients with sepsis and septic mice. Following sepsis and secondary intrapulmonary bacterial challenge, IL-27R KO mice had higher survival rates and improved bacterial clearance from lung and blood compared with WT mice, which was associated with early increased pulmonary cytokine/ chemokine production, as well as enhanced neutrophil recruitment to airspaces. Neutralisation of IL-27 in septic mice significantly improved survival and clearance of bacteria from the lungs of septic mice infected with $P$ aeruginosa, and direct application of recombinant IL-27 could increase susceptibility to $P$ aeruginosa infection. The resistance of septic IL-27R KO mice to secondary $P$ aeruginosa infection was abrogated by depletion of alveolar macrophages (AMs) and neutrophils. AMs from septic IL-27R KO mice had higher bacterial uptake and killing capacities, enhanced cytokine/chemokine production, and increased expression of costimulatory molecules compared with those from WT mice, while neutrophils from septic IL-27R KO mice had increased bacterial killing ability and higher expression of adhesion molecule Mac-1 compared with WT neutrophils.

Conclusions IL-27 is an important mediator of sepsisinduced impairment of lung antibacterial host defence.

\section{INTRODUCTION}

Sepsis is the leading cause of death in hospitalised patients. It is estimated that $15-19$ million sepsis cases occur worldwide per year with mortality rates of $20-50 \% .{ }^{12}$ Sepsis initiates a complex immunological response characterised by an intensive inflammatory response, and then the body mounts an anti-inflammatory response. ${ }^{3}$ With contemporary standard-of-care measures, most patients survive the early hyperinflammatory phase but enter a stage of immunosuppression, ${ }^{4}$ which is evidenced by the frequent occurrence of secondary nosocomial

\section{Key messages}

What is the key question?

- Does interleukin (IL)-27 play an immunopathological role in sepsis-induced secondary bacterial pneumonia?

What is the bottom line?

- $\mathrm{IL}-27 \mathrm{R}^{-1-}$ mice were more resistant to secondary bacterial pneumonia in the septic setting, with strikingly lower lung and blood bacterial burdens and increased survival compared with wild-type mice

Why read on?

- Immunomodulation of IL-27 has potential as immunotherapy during sepsis-induced secondary bacterial infections.

infections, in particular bacterial pneumonia caused by Pseudomonas aeruginosa. ${ }^{5}$

Although the potential mechanisms of immunosuppression are not fully characterised, increased interleukin (IL)-10 and decreased cell-surface antigen-presenting complex human leukocyte antigen (HLA)-DR have been implicated in increased susceptibility to secondary nosocomial infections in sepsis. ${ }^{6} 7$ In addition, increased expression of negative costimulatory molecules programmed death 1 (PD-1), cytotoxic T-lymphocyte-associated antigen 4 (CTLA-4), and B-lymphocyte and T-lymphocyte attenuator was also considered to mediate sepsis-induced immunosuppression. ${ }^{8-10}$ Importantly, some encouraging results from use of the new immunotherapeutic agent against PD-1 point the way for immunotherapy in sepsis. ${ }^{78} 11$ Identification of immunomodulatory molecules mediating sepsis-induced immunosuppression would provide a new therapeutic approach, thereby reversing the immunocompromised state and decreasing the occurrence of secondary nosocomial infections in patients with sepsis.

IL-27 is a heterodimeric cytokine composed of IL-27p28 and Epstein-Barr virus induced gene 3 (EBI3), ${ }^{12}$ which signals through a heterodimeric receptor complex consisting of the common IL-6 receptor chain gp130 and a unique IL-27 receptor $\alpha$ chain (IL-27R) WSX-1. ${ }^{13}$ IL-27 has been identified as a vital modulator of immune responses in 
Figure 1 Elevated expression of interleukin (IL)-27p28 and Epstein-Barr virus induced gene 3 (EBI3) mRNA and IL-27p28 protein in sepsis patients. (A) mRNA expression levels for IL-27p28 and EBI3 mRNA were measured by real-time PCR in whole blood obtained from patients with sepsis and healthy control donors. (B) IL-27 protein was measured by ELISA in serum samples from patients with sepsis and healthy control donors. Each symbol represents an individual subject, and horizontal bars represent median values. Data were analysed by Mann-Whitney test, ${ }^{* *} p<0.01,{ }^{* * *} p<0.001$ when compared between groups denoted by horizontal lines.
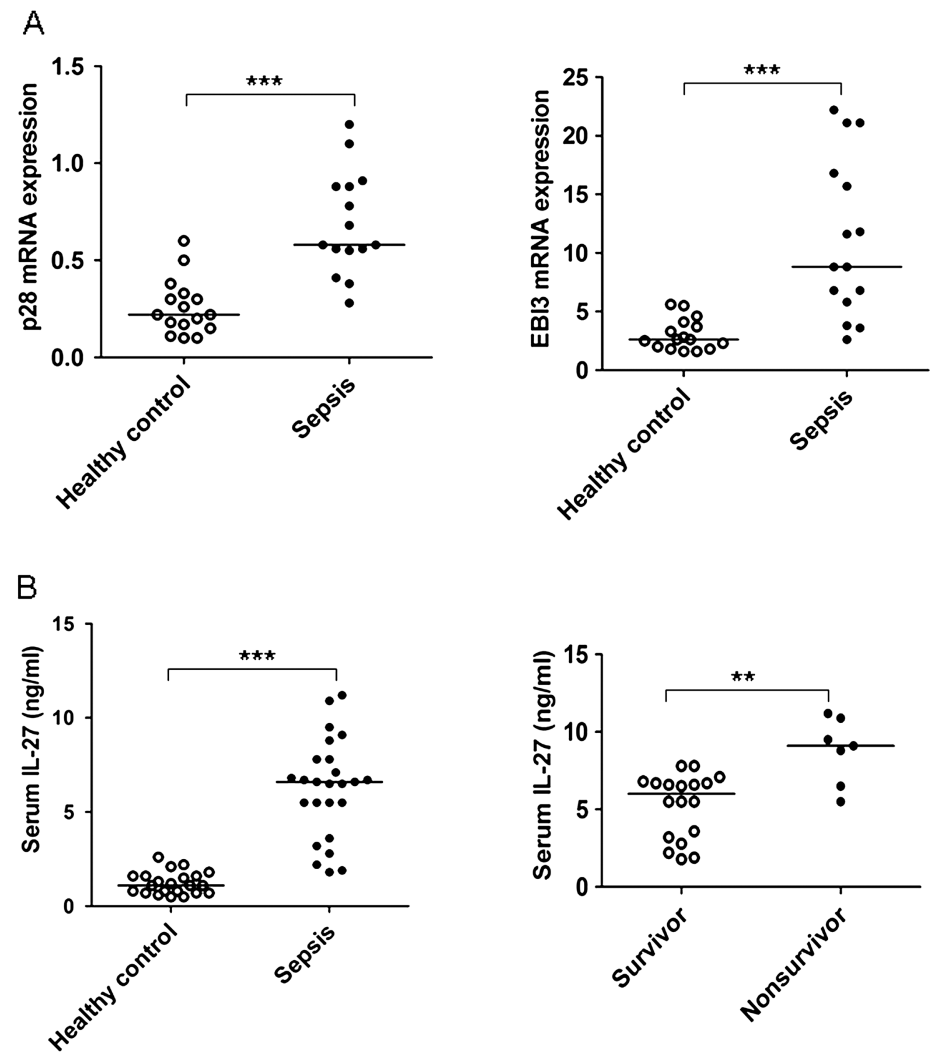
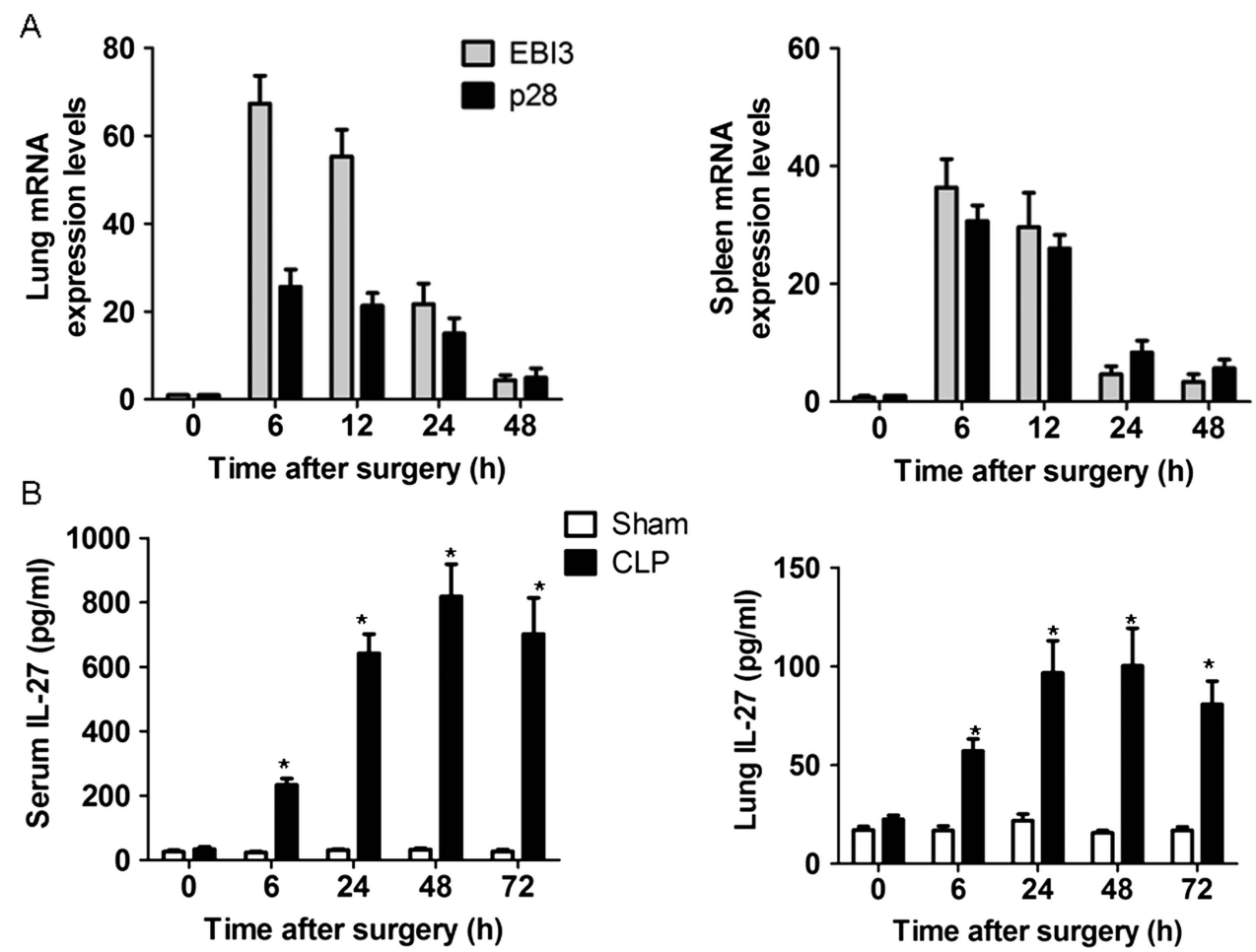

Figure 2 Increased expression levels of interleukin (IL)-27 in septic mice. (A) Wild-type mice were subjected to sham or caecal ligation and puncture (CLP) surgery. Lungs and spleens were removed for total RNA isolation at the indicated time points and quantitative real-time PCR was performed. Mean values are shown as fold induction relative to transcript levels in mice at $0 \mathrm{~h}$ ( $\mathrm{n}=6$ mice/group). (B) Blood and lungs were obtained from mice at the indicated time points after sham or CLP surgery. Samples were assayed for IL-27 content by specific ELISA ( $n=6$ mice/group). Data were expressed as mean \pm SEM and analysed using Mann-Whitney test, ${ }^{*} \mathrm{p}<0.05$ when compared with sham mice. EBI3, Epstein-Barr virus induced gene 3 . 

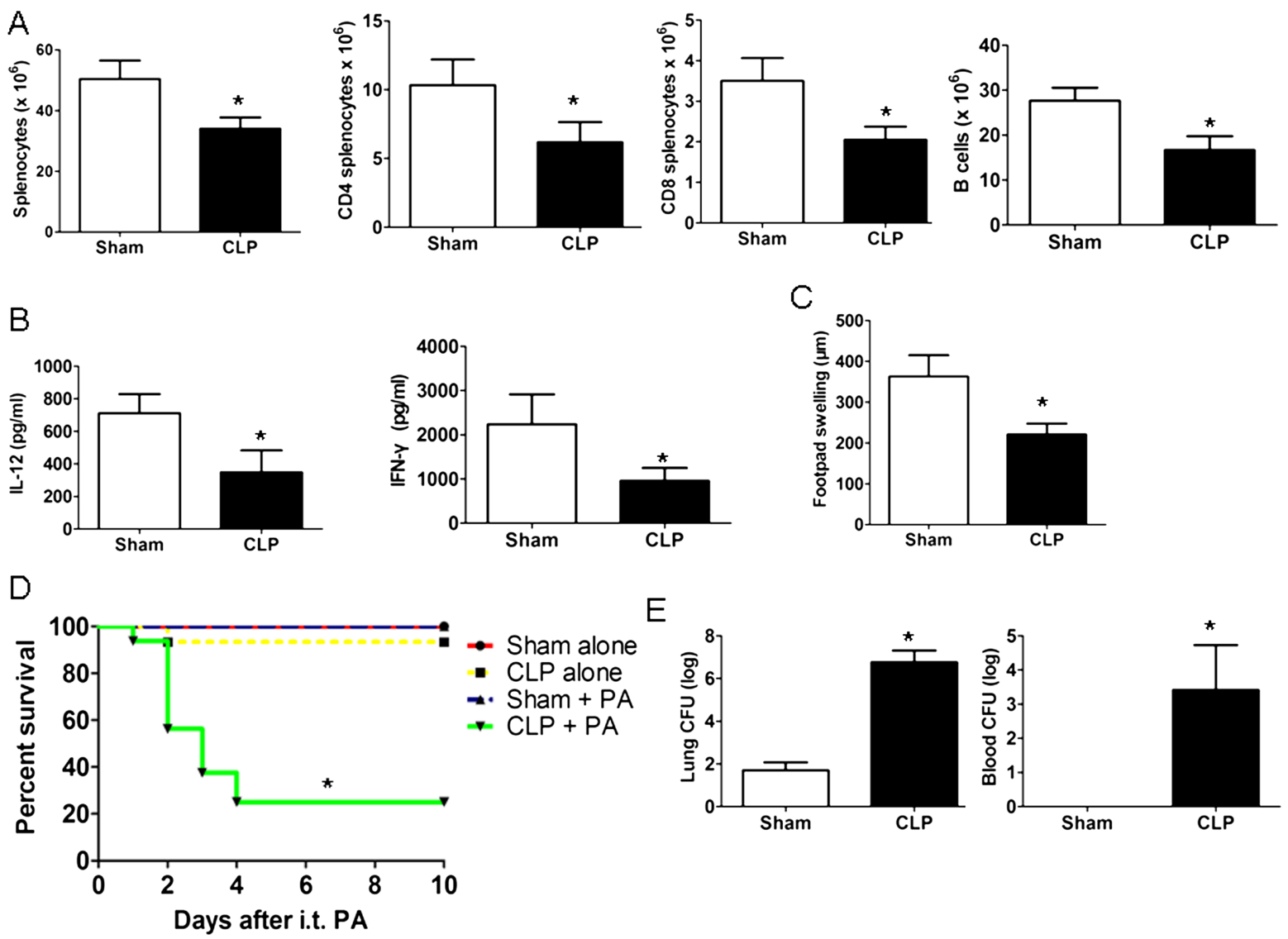

$\mathrm{E}$
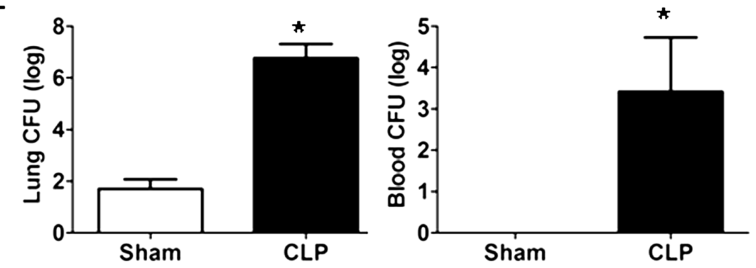

Days after i.t. PA

Figure 3 Caecal ligation and puncture (CLP) resulted in impaired host immune responses. (A) Viable splenocyte subsets were quantitated by flow cytometry $24 \mathrm{~h}$ after surgery in sham and CLP mice ( $\mathrm{n}=6$ mice/group). (B) Splenocytes isolated from sham or CLP mice were stimulated with heat-killed (HK) Pseudomonas aeruginosa (equivalent to $1 \times 10^{6}$ colony-forming units (CFUs) per $\mathrm{mL}$ ) for $24 \mathrm{~h}$, after which IL-12 and interferon $\gamma($ IFN- $\gamma$ ) were determined by ELISA ( $n=6$ mice/group). (C) Mice underwent sham or CLP surgery; 4 days post surgery, mice were sensitised with trinitrophenol (TNP), and 4 days after sensitisation, mice had rechallenge with TNP (footpad injection). At $24 \mathrm{~h}$ after rechallenge, the degree of footpad swelling was quantitated versus phosphate-buffered saline (PBS) injection ( $n=6$ mice/group). (D) Wild-type mice underwent either CLP or sham surgery; $24 \mathrm{~h}$ later, mice were administered intratracheal $P$ aeruginosa and monitored for 10 days after challenge for survival ( $\mathrm{n}=16$ mice/ group). (E) Lung and blood CFUs in sham and CLP mice at $24 \mathrm{~h}$ after secondary $P$ aeruginosa infection ( $\mathrm{n}=6$ mice/group). Survival curves were analysed using the log-rank (Mantel-Cox) test, and other data were expressed as mean \pm SEM and analysed using the Mann-Whitney test. * $p<0.05$ compared with sham mice. i.t., intratracheal; PA, $P$ aeruginosa.

T cells, ${ }^{12} 1415$ and it also regulates biological functions of other immune effector cells, such as macrophages and neurtophils. $^{13} 1617$

Regarding the regulatory role of IL-27 in host immune responses, we hypothesised that IL-27 may participate in sepsis-induced immunosuppression. In this study, we sought to investigate the effects of IL-27 on pulmonary host defence against secondary $P$ aeruginosa pneumonia in a model of caecal ligation and puncture (CLP)-induced sepsis.

\section{METHODS}

See online supplement for additional details.

\section{Study population}

Patients were randomly selected from The First Affiliated Hospital of Chongqing Medical University intensive care unit. The diagnosis of sepsis was based on the criteria recommended by the American College of Chest Physicians and Society of Critical Care Medicine Consensus Conference. ${ }^{18}$ The study was approved by the Clinical Research Ethics Committee of The First Affiliated Hospital of Chongqing Medical University.

\section{Animal models}

CLP was used as a model of systemic sepsis syndrome. ${ }^{19}$ To create a relevant model of secondary pneumonia, $1 \times 10^{5} P$ aeruginosa (strain UI-18) was administered intratracheally to CLP or sham mice.

\section{Statistics}

Data were expressed as mean \pm SEM. Differences between groups were analysed by Mann-Whitney test or one-way analysis of variance (ANOVA) with Bonferroni's multiple comparison post hoc tests if appropriate. Survival curves were compared using the log-rank (Mantel-Cox) test. A p value of 0.05 or less was considered statistically significant. GraphPad Prism V.5 software was used for all statistical analysis and graphing.

\section{RESULTS}

IL-27 is elevated in patients with sepsis

To study whether IL-27 is expressed in sepsis, we detected IL-27 expression levels in whole blood collected from a cohort of patients with sepsis. The demographic and clinical characteristics of the subjects are listed in online supplementary table S1. Quantitative real-time PCR analysis 

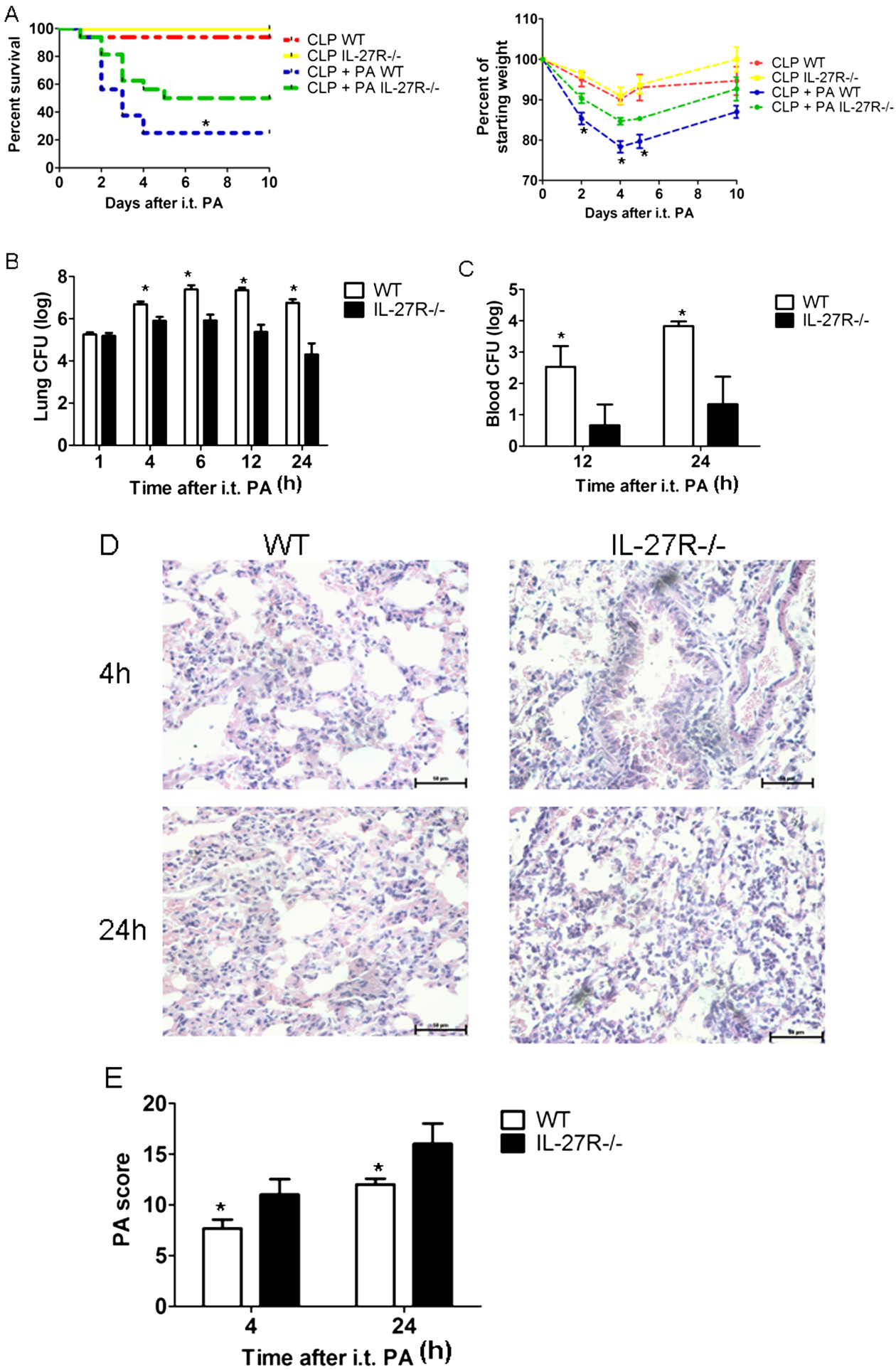

Figure 4 Septic interleukin (IL)-27R $\mathrm{R}^{-1-}$ mice were resistant to secondary Pseudomonas aeruginosa pneumonia. (A) Wild-type (WT) and IL-27R ${ }^{-/-}$ mice underwent either caecal ligation and puncture (CLP) or sham surgery; $24 \mathrm{~h}$ later, mice were administered intratracheal $P$ aeruginosa and monitored for survival and body weight relative to baseline over a 10-day period after challenge ( $n=16$ mice/group). (B and C) Clearance of $P$ aeruginosa by septic WT and IL-27R ${ }^{-1-}$ mice. Lungs (B) and blood (C) were harvested at the indicated time points following secondary intratracheal $P$ aeruginosa infection after CLP, for assessment of colony-forming units (CFUs) ( $n=6$ mice/group). (D) Representative H\&E stainings of lung tissue at 4 or $24 \mathrm{~h}$ after secondary inoculation with $P$ aeruginosa in WT and IL-27R ${ }^{-1-}$ mice. (E) Histological scores of secondary $P$ aeruginosa pneumonia in septic WT and IL-27R ${ }^{-1-}$ mice ( $n=6$ mice/group). Survival curves were analysed using the log-rank (Mantel-Cox) test, and other data were expressed as mean \pm SEM and analysed using the non-parametric Mann-Whitney test. ${ }^{*} \mathrm{p}<0.05$ compared with IL-27R ${ }^{-1-}$ mice. i.t., intratracheal; PA, $P$ aeruginosa.

showed that expression of EBI3 and IL-27p28 subunits was markedly elevated in patients with sepsis compared with that of healthy control subjects (figure 1A). In addition, IL-27 protein levels in serum samples of patients with sepsis were significantly higher than those of healthy individuals (figure 1B). Notably, those who did not survive had significantly more 
serum IL-27 than the survivors (figure 1B). Thus, IL-27 may play a major part in clinical sepsis.

\section{CLP induces elevated IL-27 expression}

We next examined the expression of IL-27 in the murine CLP model. Accordingly, we analysed the expression of EBI3 and p28 subunits of IL-27 in the lung and spleen during the course of sepsis. Six hours after CLP, IL-27 expression in these tissues increased and reached a maximum level at $12 \mathrm{~h}$, then declined thereafter (figure 2A). IL-27-specific ELISA further confirmed that IL-27 protein was upregulated in the blood and lung $6 \mathrm{~h}$ after CLP, which peaked at $48 \mathrm{~h}$ and then declined (figure 2B).

\section{CLP results in a defect in immune function of wild-type mice}

To determine the changes in host immunity following CLP, cellular components of the spleen were first quantified. Compared with sham mice, CLP caused a significant loss in absolute cell counts for total splenocytes, CD4 T, CD T and $\mathrm{B}$ cells (figure 3A). Splenocytes from CLP mice also displayed significantly decreased levels of IL-12 and interferon (IFN)- $\gamma$ compared with those from sham mice (figure 3B). In addition, CLP mice had an impaired delayed-type hypersensitivity response compared with sham mice (figure $3 \mathrm{C}$ ). Furthermore, CLP using a 26-gauge puncture led to $0-10 \%$ mortality in wildtype (WT) mice from three independent experiments (data not shown), which was indistinguishable from sham mice. In contrast, CLP mice had substantial mortality when subsequently challenged with $P$ aeruginosa (figure $3 \mathrm{D}$ ). This high lethality was associated with significantly increased $P$ aeruginosa colonyforming units (CFUs) in the lung and blood of CLP mice compared with sham mice (figure $3 \mathrm{E}$ ). These data are in accordance with earlier investigations and show that CLP impairs the host immune responses, resulting in increased susceptibility to secondary bacterial pneumonia. ${ }^{3} 6$

\section{Survival of WT and WSX-1-deficient (IL-27R ${ }^{-1-}$ ) mice following CLP and subsequent secondary pneumonia with $P$ aeruginosa}

Regarding the increased expression of IL-27 during sepsis, we hypothesised that IL-27 may play an important role in the pathophysiology of sepsis. However, the mortality of IL-27R $\mathrm{R}^{-/-}$ mice was not significantly different from that of their WT counterparts following CLP alone (figure 4A). Since CLP resulted in the impairment of immune response, we investigated the role of IL-27 in host defence against secondary $P$ aeruginosa challenge in septic mice. IL-27R $\mathrm{R}^{-/}$mice showed significantly decreased mortality and weight loss after secondary pulmonary $P$ aeruginosa infection compared with WT mice in the septic setting (figure 4A). This decreased mortality was associated with controlled bacterial growth in the lung (figure 4B) and in the peripheral blood (figure $4 \mathrm{C}$ ). H\&E staining of lung sections showed that lung inflammation was exaggerated in IL-27R $\mathrm{R}^{-/-}$ mice at 4 or $24 \mathrm{~h}$ after induction of secondary $P$ aeruginosa infection (figure 4D), which was further reflected by significantly higher pathology scores compared with WT mice (figure 4E). These data suggest that IL-27R deficiency confers protection against secondary pneumonia with $P$ aeruginosa in septic mice.

\section{Lung cytokine production in septic WT and IL-27R $\mathrm{R}^{-I-}$ mice following secondary $P$ aeruginosa infection}

To determine underlying mechanisms by which IL-27R $\mathrm{R}^{-/-}$mice were protected from lethality by secondary challenge with $P$
Table 1 Total lung cytokine levels $(\mathrm{pg} / \mathrm{mL})$ in septic wild-type (WT) and interleukin (IL)-27R-I- mice after secondary challenge with Pseudomonas aeruginosa

\begin{tabular}{|c|c|c|c|}
\hline & 0 & $4 \mathrm{~h}$ & $24 \mathrm{~h}$ \\
\hline \multicolumn{4}{|l|}{ TNF- $\alpha$} \\
\hline WT & $188 \pm 56$ & $333 \pm 85$ & $521 \pm 76$ \\
\hline IL-27R-I- & $211 \pm 87$ & $581 \pm 161$ * & $663 \pm 131$ \\
\hline \multicolumn{4}{|l|}{ IL-1 $\beta$} \\
\hline WT & $133 \pm 59$ & $253 \pm 81$ & $356 \pm 113$ \\
\hline IL-27R-I- & $226 \pm 88$ & $468 \pm 116^{*}$ & $612 \pm 176$ \\
\hline \multicolumn{4}{|l|}{ CXCL1 } \\
\hline WT & $372 \pm 112$ & $551 \pm 162$ & $761 \pm 176$ \\
\hline IL-27R-I- & $486 \pm 98$ & $887 \pm 152$ * & $826 \pm 168$ \\
\hline \multicolumn{4}{|l|}{ CXCL2 } \\
\hline WT & $121 \pm 57$ & $281 \pm 76$ & $316 \pm 103$ \\
\hline IL-27R-I- & $176 \pm 85$ & $423 \pm 100$ * & $488 \pm 176$ \\
\hline \multicolumn{4}{|l|}{ CXCL10 } \\
\hline WT & $622 \pm 137$ & $1008 \pm 515$ & $1536 \pm 656$ \\
\hline IL-27R-I- & $867 \pm 226$ & $1883 \pm 917^{*}$ & $2127 \pm 1168$ * \\
\hline \multicolumn{4}{|l|}{ IL-6 } \\
\hline WT & $512 \pm 167$ & $778 \pm 333$ & $1179 \pm 613$ \\
\hline IL-27R-I- & $692 \pm 328$ & $1097 \pm 512^{*}$ & $1088 \pm 675$ \\
\hline \multicolumn{4}{|l|}{ IL-12 } \\
\hline WT & $311 \pm 112$ & $453 \pm 184$ & $583 \pm 222$ \\
\hline IL-27R-I- & $466 \pm 176$ & $727 \pm 226$ * & $813 \pm 336$ \\
\hline \multicolumn{4}{|l|}{ IL-17 } \\
\hline WT & $115 \pm 55$ & $279 \pm 165$ & $421 \pm 126$ \\
\hline IL-27R-I- & $176 \pm 73$ & $511 \pm 183^{*}$ & $633 \pm 262$ \\
\hline \multicolumn{4}{|l|}{ IL-10 } \\
\hline WT & $1768 \pm 843$ & $3611 \pm 1347$ & $4611 \pm 1356$ \\
\hline IL-27R-I- & $1255 \pm 933$ & $2151 \pm 1687$ & $2873 \pm 1634^{*}$ \\
\hline
\end{tabular}

Data were expressed as mean \pm SEM. Differences between WT and IL-27R-I- mice were analysed using the non-parametric Mann-Whitney test.

${ }^{*} p<0.05$ compared with corresponding WT control. $n=6$ mice per time point.

TNF- $\alpha$, tumour necrosis factor $\alpha$

aeruginosa, we first examined the influence of IL-27R deficiency on the induction of cytokine/chemokine in the lung. As shown in table 1 , IL-27R $\mathrm{R}^{-/-}$mice had significantly higher levels of tumour necrosis factor $\alpha$ (TNF- $\alpha)$, IL-1 $\beta$, CXCL1, CXCL2, CXCL10, IL-6, IL-12 and IL-17 at the early time point (4 h after $P$ aeruginosa administration) compared with infected WT mice, and levels of CXCL10 remained significantly elevated in IL-27R $\mathrm{R}^{-/-}$mice even at $24 \mathrm{~h}$. In addition, septic IL-27R ${ }^{-1-}$ mice

Table 2 Lung cell counts in septic wild-type (WT) and interleukin (IL)-27R-I- mice after challenge with Pseudomonas aeruginosa

\begin{tabular}{llll}
\hline & Monocytes & Neutrophils & Lymphocytes \\
\hline $4 \mathrm{~h}$ & & & \\
WT & $6.8 \times 10^{5} \pm 1.1 \times 10^{5}$ & $1.5 \times 10^{5} \pm 1.2 \times 10^{5}$ & $0.9 \times 10^{4} \pm 0.8 \times 10^{4}$ \\
$\quad$ IL-27R-I- & $7.1 \times 10^{5} \pm 1.3 \times 10^{5}$ & $3.4 \times 10^{5} \pm 1.6 \times 10^{5 *}$ & $1.1 \times 10^{4} \pm 1.2 \times 10^{4}$ \\
$24 \mathrm{~h}$ & & & \\
WT & $7.6 \times 10^{5} \pm 1.5 \times 10^{5}$ & $2.6 \times 10^{5} \pm 1.6 \times 10^{5}$ & $1.1 \times 10^{4} \pm 0.5 \times 10^{4}$ \\
IL-27R-I- & $7.9 \times 10^{5} \pm 1.7 \times 10^{5}$ & $4.3 \times 10^{5} \pm 2.3 \times 10^{5 *}$ & $0.7 \times 10^{4} \pm 0.7 \times 10^{4}$
\end{tabular}

Data were expressed as mean \pm SEM. Differences between WT and IL-27R-I- mice were analysed using the non-parametric Mann-Whitney test.

${ }^{*} p<0.05$ compared with corresponding WT control. $n=6$ mice per time point. 
Figure 5 Interleukin (IL)-27

neutralisation restored pulmonary host defence in septic wild-type (WT) mice.

(A) Survival of septic WT mice following IL-27 neutralisation with anti-IL-27 blocking antibodies upon secondary Pseudomonas aeruginosa infection ( $\mathrm{n}=16$ mice/group). ( $\mathrm{B}$ and $\mathrm{C}$ ) Lung and blood colony-forming units (CFUs) in septic WT mice following IL-27 neutralisation with anti-IL-27 blocking antibodies upon secondary $P$ aeruginosa infection ( $\mathrm{n}=6$ mice/group). (D) The release of cytokine/chemokine in the lungs of septic WT mice following IL-27 neutralisation with anti-IL-27 blocking antibodies at $4 \mathrm{~h}$ upon secondary $P$ aeruginosa infection ( $n=6$ mice/group). (E) The number of neutrophils in the lungs at $4 \mathrm{~h}$ after secondary $P$ aeruginosa challenge ( $n=6$ mice/group). Survival curves were analysed using the log-rank (Mantel-Cox) test, and other data were expressed as mean \pm SEM and analysed using the non-parametric Mann-Whitney test. * $p<0.05$ compared with mice treated with anti-IL-27 antibodies. i.t., intratracheal; PA, $P$ aeruginosa; TNF- $\alpha$, tumour necrosis factor $\alpha$.

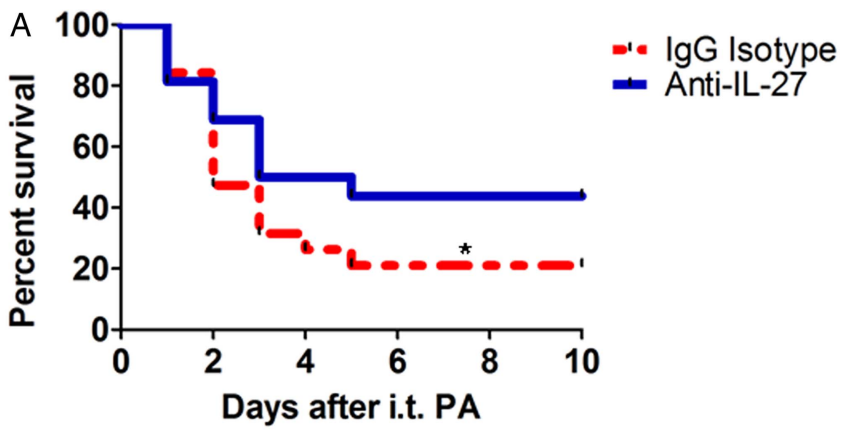

B

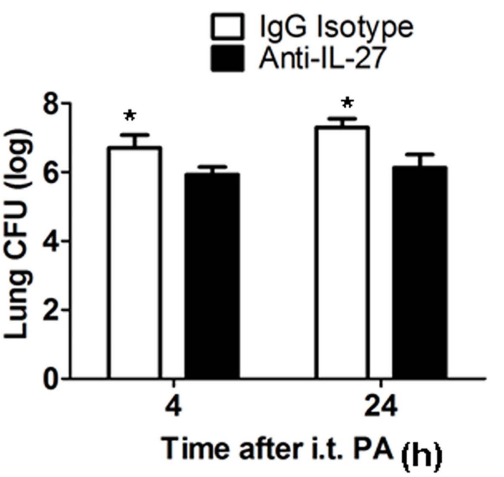

C

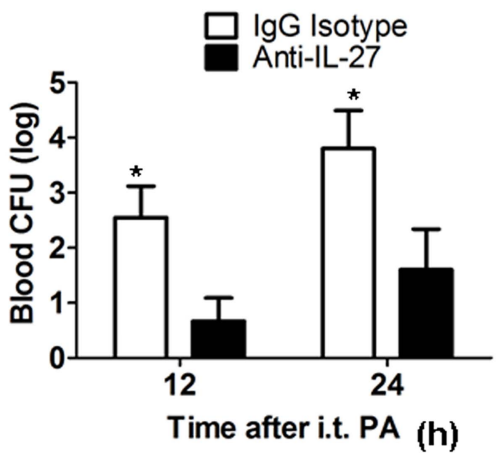

$\mathrm{D}$
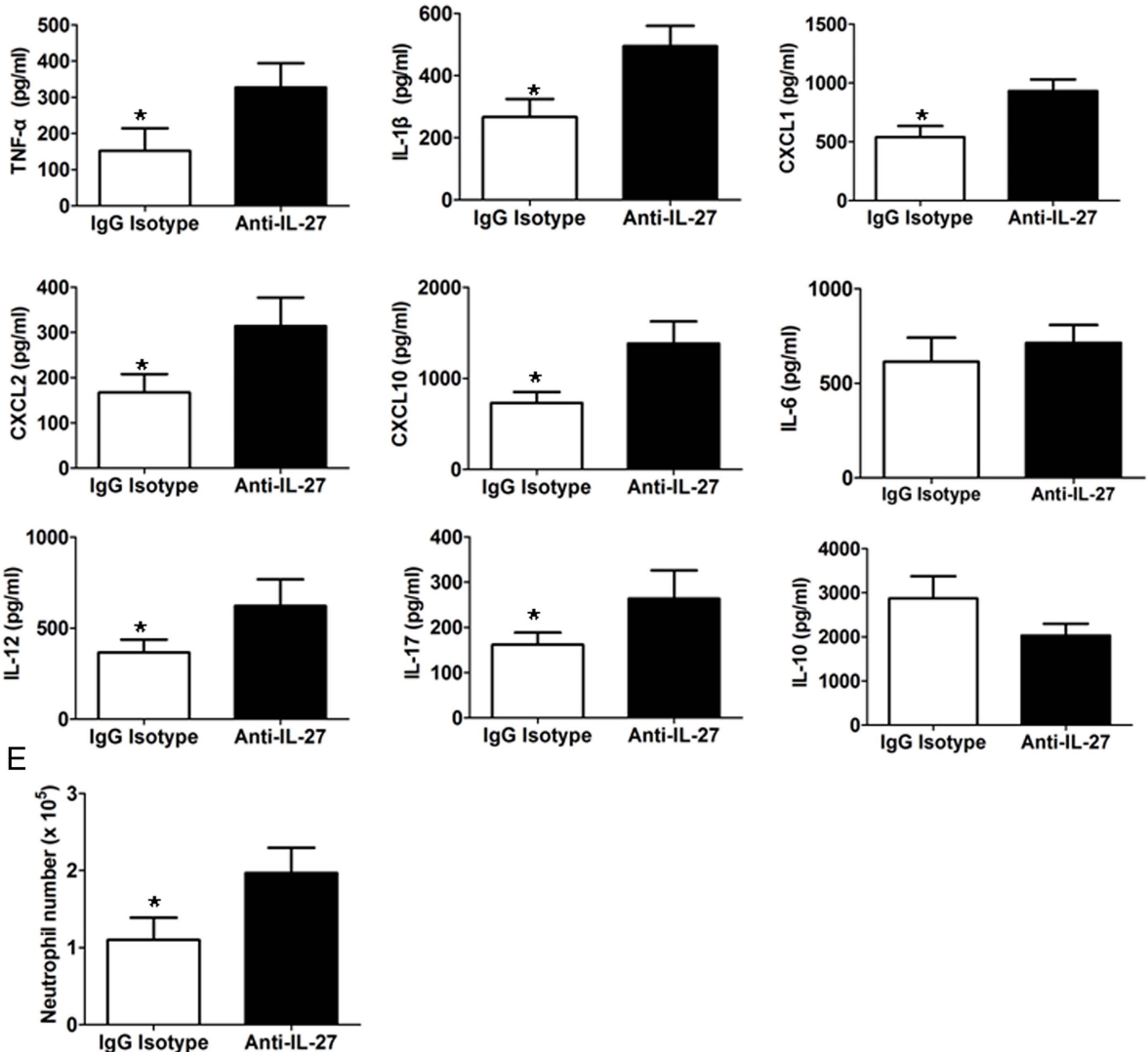

displayed a significantly lower concentration of antiinflammatory cytokine IL-10 at $24 \mathrm{~h}$ after secondary $P$ aeruginosa infection. These findings were consistent with the increased lung inflammation in IL-27 $\mathrm{R}^{-/-}$mice (figure 4D), and suggest that IL-27R $\mathrm{R}^{-1-}$ deficiency skews the cytokine balance towards an early proinflammatory response that may promote bacterial clearance.
Lung leukocyte recruitment in WT and IL-27R ${ }^{-1-}$ mice following secondary $P$ aeruginosa infection

Having observed the significant difference in cytokine/chemokine production, we further assessed lung recruitment of leukocytes 4 and $24 \mathrm{~h}$ after $P$ aeruginosa administration in septic WT and IL-27R $\mathrm{R}^{-/-}$mice. Intriguingly, at 4 and $24 \mathrm{~h}$ following intratracheal $P$ aeruginosa infection, septic IL-27R $\mathrm{R}^{-/-}$mice had a 
significantly higher number of neutrophils in the lungs compared with septic WT mice (table 2). However, there was no significant difference in the number of monocytes and lymphocytes.

\section{Depletion of IL-27 reduces the susceptibility to secondary $P$ aeruginosa infection}

Because septic IL-27R $\mathrm{R}^{-/-}$mice were protected from secondary $P$ aeruginosa infection, we determined the potential beneficial effects of blocking the biological function of IL-27 in vivo. Septic WT mice were pretreated with anti-IL-27 neutralising antibodies and then infected with $P$ aeruginosa. Anti-IL-27-treated mice had significantly higher survival (figure 5A) and enhanced clearance of $P$ aeruginosa from the lung (figure 5B) and blood (figure 5C) compared with control IgG-treated mice. In addition, treatment with anti-IL-27 antibodies increased the early levels of cytokines and chemokines (except for IL-10), and the early numbers of neutrophils in the lung of septic mice (figure $5 \mathrm{E}, \mathrm{F}$ ). These results are similar to what was observed in IL-27R $\mathrm{R}^{-/-}$mice.

\section{Treatment with recombinant IL-27 promotes development of $P$ aeruginosa pneumonia}

To directly determine the influence of IL-27, we inoculated it into naïve mice and then mice were subjected to $P$ aeruginosa infection. Treatment with recombinant IL-27 significantly increased mortality (figure 6A) and deceased bacterial clearance from the lung (figure 6B) and blood (figure 6C) compared with control mice. IL-27 treatment also reduced the early levels of cytokines and chemokines (except for IL-10) and the early numbers of neutrophils in the lung (figure $6 \mathrm{E}, \mathrm{F}$ ), suggesting that inoculation of exogenous IL-27 mimics sepsis settings.

\section{IL-27R deficiency modulates functions of alveolar macrophages}

Alveolar macrophages (AMs) constitute up to $95 \%$ of the immune cells in the alveolar space, and they are the first line of host defence against bacteria in the lung. Besides their scavenger functions, such as phagocytosis and subsequent digestion of bacteria, AMs can promote the generation of subsequent inflammatory responses by expressing cytokines, chemokines or other inflammatory mediators. ${ }^{20-22}$ As shown in figure 7 , depletion of AMs significantly increased mortality (figure 7A) and deceased bacterial clearance from the lung (figure 7B) and blood (figure 7C) in septic IL-27R $\mathrm{R}^{-1-}$ mice upon secondary $P$ aeruginosa infection. We therefore investigated the contribution of IL-27 to the responsiveness of AMs in sepsis. CLP resulted in a significant increase in IL-27R mRNA in pulmonary macrophages (alveolar plus interstitial) compared with sham controls (figure 7D). To exclude the influence of interstitial macrophages on the mRNA expression of IL-27R detected by real-time PCR, we next examined IL-27R protein expression on the cell surface of AMs, and IL-27R were expressed at higher levels on AMs lavaged from septic mice compared with sham controls (figure 7E). Then we studied bacterial uptake and killing capacities of AMs. Bacterial uptake and killing capacities were significantly higher in AMs isolated from septic IL-27 $\mathrm{R}^{-/-}$mice compared with those from WT mice (figure 7F), and treatment with recombinant IL-27 inhibited bacterial uptake and killing capacities in AMs from WT mice but not IL-27R $\mathrm{R}^{-/-}$mice.

To further assess the activation status of AMs during secondary infection, we determined the expression of cytokines, chemokines and cell-surface costimulatory molecules by AMs after CLP upon stimulation with heat-killed $P$ aeruginosa. AMs from IL-27R $\mathrm{R}^{-/}$mice showed significantly higher production of TNF- $\alpha$, IL-1 $\beta$, CXCL1, CXCL2, CXCL10, IL-6 and IL-12 than those from WT mice (see online supplementary figure S1A). Treatment with recombinant IL-27 inhibited the production of
Figure 6 Interleukin (IL)-27 treatment impaired pulmonary host immunity in wild-type (WT) mice. WT mice were injected with intraperitoneal recombinant IL-27 or saline, and then mice were challenged with intraperitoneal Pseudomonas aeruginosa $24 \mathrm{~h}$ later. (A) Survival of WT mice treated with recombinant IL-27 upon $P$ aeruginosa infection ( $n=12$ mice/group). (B and C) Lung and blood colony-forming units (CFUs) in WT mice treated with recombinant IL-27 upon $P$ aeruginosa infection $(n=6$ mice/group). (D) The release of cytokine/chemokine in the lungs of WT mice treated with recombinant IL-27 at $4 \mathrm{~h}$ upon $P$ aeruginosa infection $(n=6$ mice/group). (E) The number of neutrophils in the lungs at $4 \mathrm{~h}$ after $P$ aeruginosa challenge ( $\mathrm{n}=6$ micel group). Survival curves were analysed using the log-rank (Mantel-Cox) test, and other data were expressed as mean \pm SEM and analysed using the non-parametric Mann-Whitney test. ${ }^{*} p<0.05$ compared with mice treated with recombinant IL-27. i.t., intratracheal; PA, $P$ aeruginosa; TNF- $\alpha$, tumour necrosis factor $\alpha$.
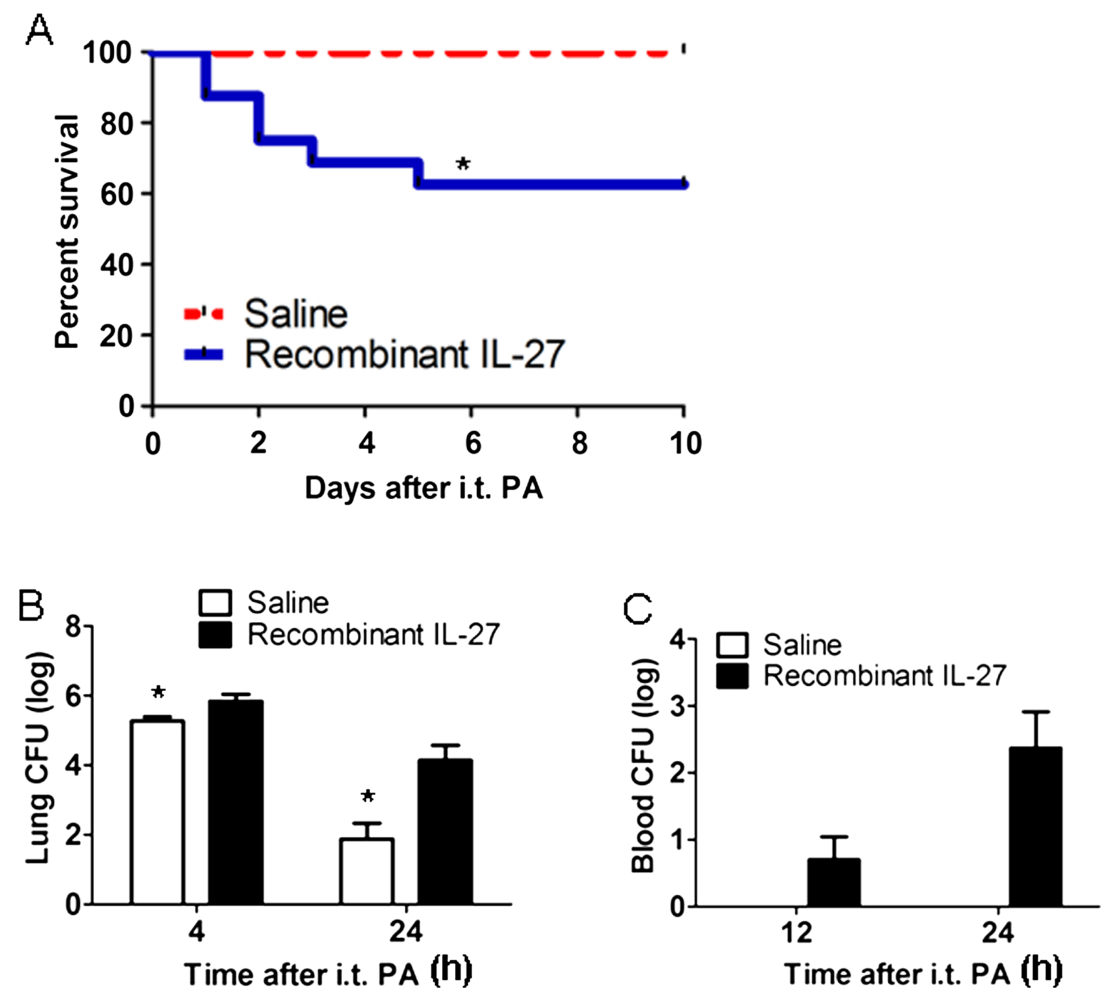
Figure 6 Continued
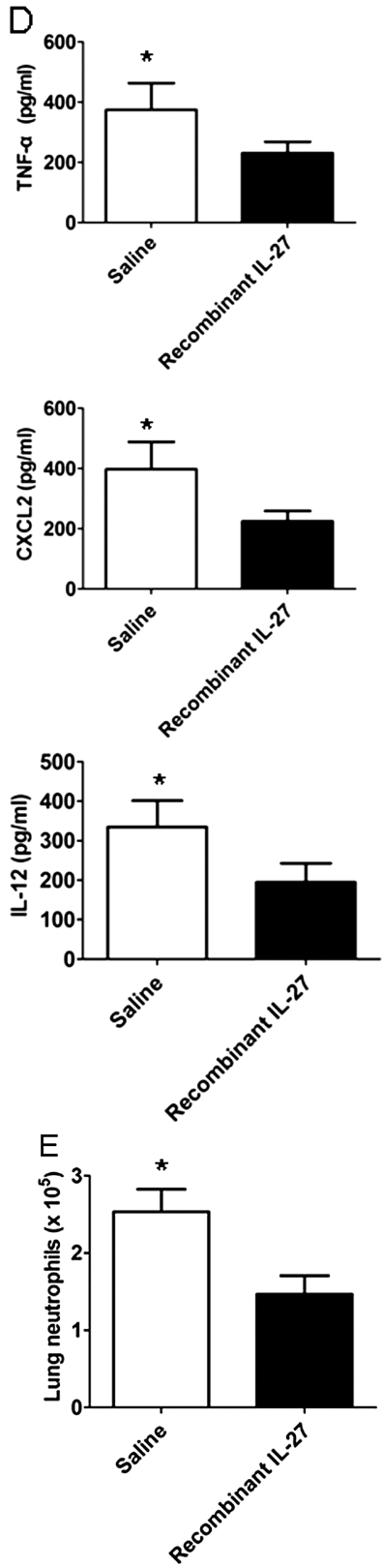
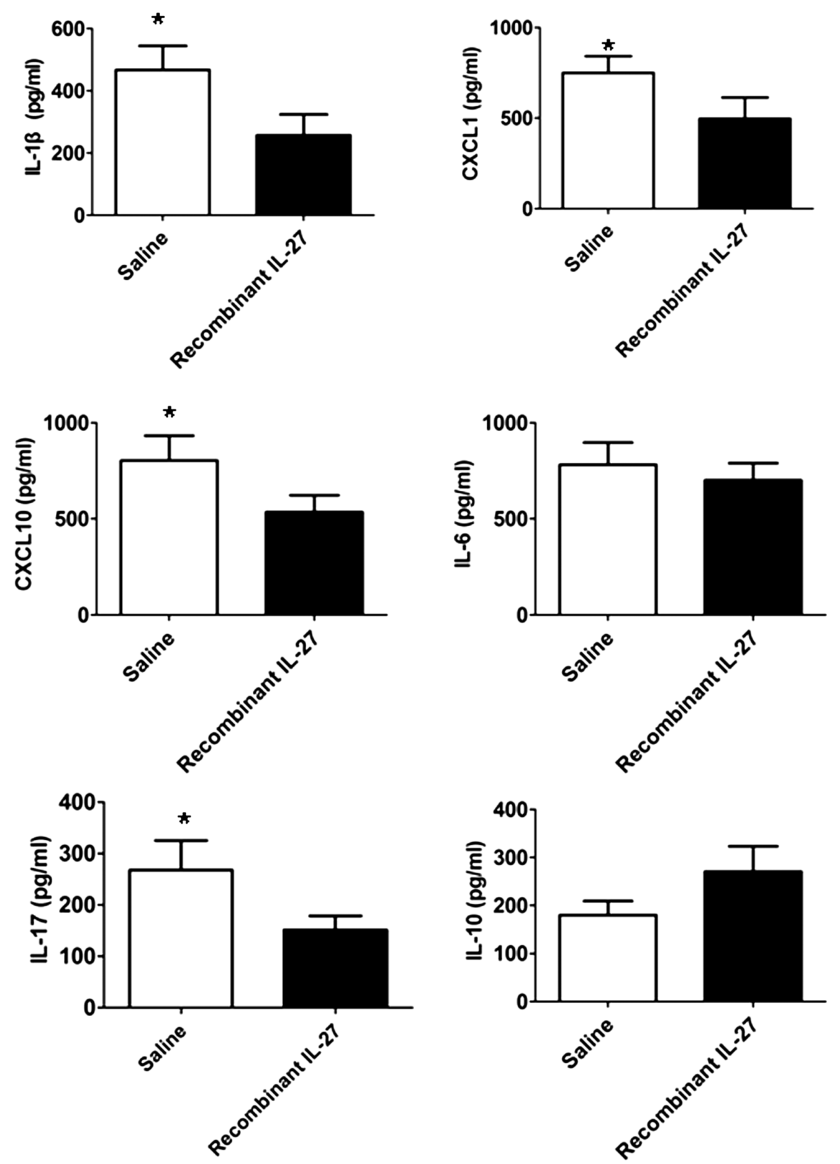

these inflammatory mediators in AMs from WT mice but not from IL-27R $\mathrm{R}^{-/-}$mice. In addition, a significant higher expression of CD40, CD86 or CD80 was noted in AMs from IL-27R $\mathrm{R}^{-/-}$mice compared with WT mice after stimulation with heat-killed $P$ aeruginosa (see online supplementary figure $\mathrm{S} 1 \mathrm{~B}$ ). Also, the addition of IL-27 resulted in a significantly decreased expression of CD40, CD86 or CD80 on WT AMs, but not IL-27R ${ }^{-/-}$AMs.

Although IL-27 has been reported to stimulate the production of anti-inflammatory cytokine IL-10, ${ }^{12}$ addition of anti-IL-10 blocking antibodies showed no apparent effect on bacterial uptake and killing capacities, and the expression of cytokine/ chemokine and cell-surface costimulatory molecules in AMs mediated by IL- 27 .

\section{IL-27R influences the responsiveness of neutrophils towards $P$ aeruginosa}

Neutrophil recruitment mediated by cytokine/chemokine from activated AMs at the site of infection is also an essential component of the early immune responses to clear invading bacteria in the lung, ${ }^{19}$ and depletion of neutrophils significantly increased mortality (figure 8A) and deceased bacterial clearance from the lung (figure $8 \mathrm{~B}$ ) and blood (figure 8C) in septic IL-27R $\mathrm{R}^{-/-}$mice upon secondary $P$ aeruginosa infection. The contribution of IL-27 to the responsiveness of neutrophils in sepsis was then investigated. We found that the mRNA level of IL-27R in neutrophils recovered from CLP mice was not significantly different from that of sham-operated mice (figure 8D), and flow cytometry analysis confirmed that the level of IL-27R protein was comparable in septic CLP and sham-operated mice (figure $8 \mathrm{E}$ ). We further investigated whether IL-27R deficiency influenced intrinsic antibacterial functions of neutrophils and found that bacterial killing ability was significantly higher in IL-27R $\mathrm{R}^{-/-}$neutrophils than that of WT neutrophils, while bacterial uptake was not significantly different between IL-27R ${ }^{-/-}$ and WT neutrophils (figure $8 \mathrm{~F}$ ). When proinflammatory cytokines were assayed in culture supernatants from neutrophils stimulated by heat-killed $P$ aeruginosa, we found that exogenous 


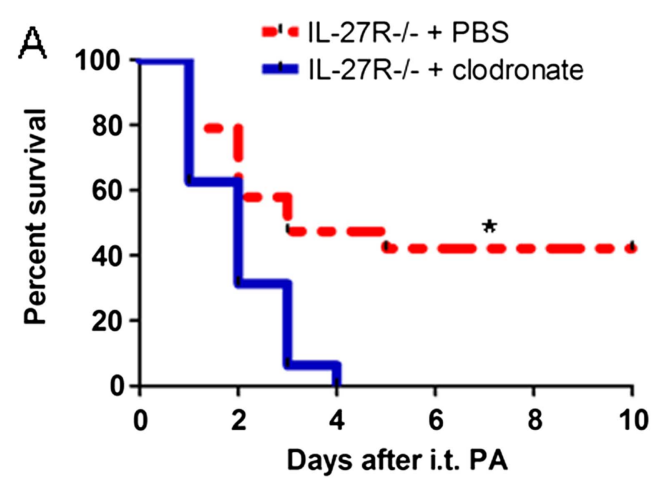

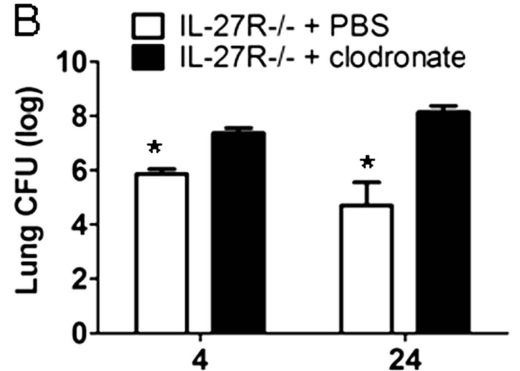

Time after i.t. PA (h)

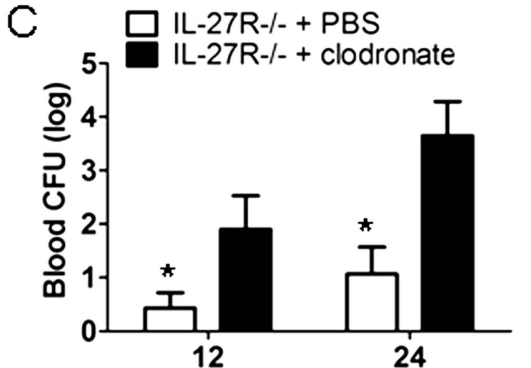

Time after i.t. PA (h)
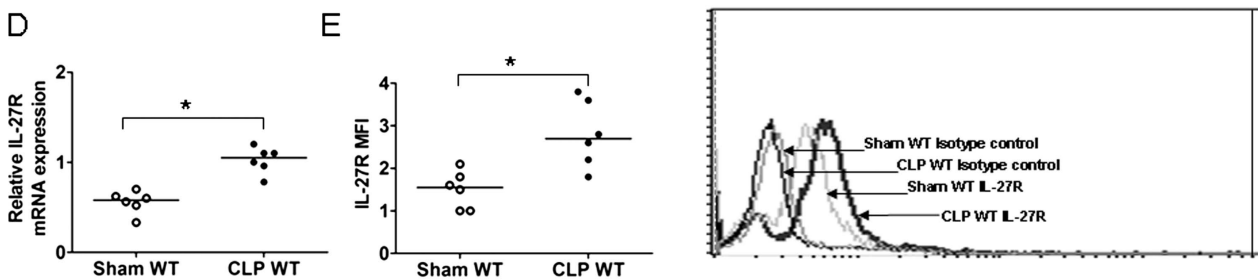

$\mathrm{F}$
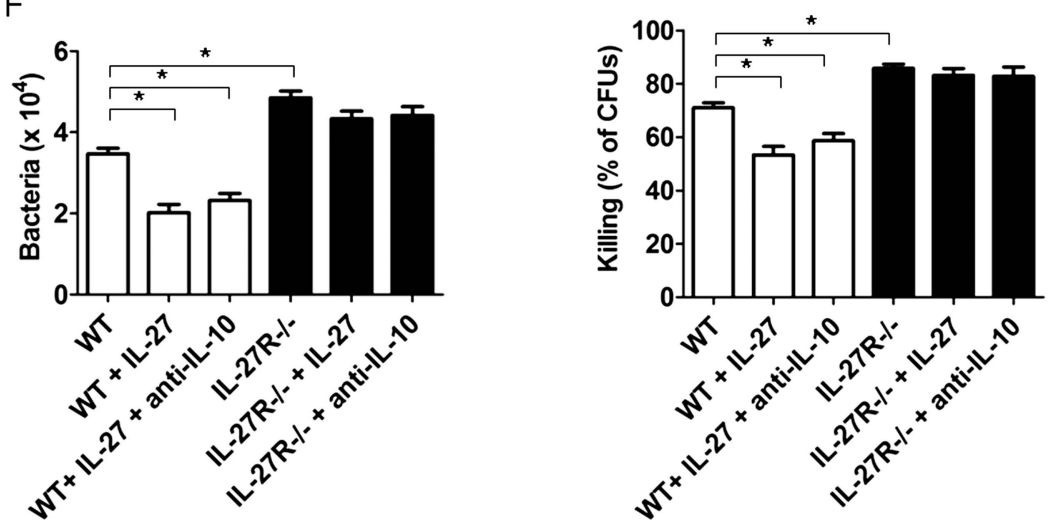

Figure 7 Interleukin (IL)-27 modulated immune responses of alveolar macrophages (AMs). (A) Survival of septic IL-27R ${ }^{-l-}$ mice depleted of AMs upon secondary Pseudomonas aeruginosa infection. IL-27R ${ }^{-1-}$ mice underwent caecal ligation and puncture (CLP) surgery; $24 \mathrm{~h}$ later, mice were given clodronate liposomes or phosphate-buffered saline (PBS) liposomes followed by secondary $P$ aeruginosa challenge $24 \mathrm{~h}$ later ( $\mathrm{n}=16$ mice/ group). Survival curves were analysed using the log-rank (Mantel-Cox) test. * $\mathrm{p}<0.05$ compared with mice treated with clodronate liposomes. (B and C) Lung and blood colony-forming units (CFUs) in IL-27R ${ }^{-1-}$ mice depleted of AMs upon secondary $P$ aeruginosa infection ( $n=6$ mice/group); data were expressed as mean $\pm S E M$ and analysed using the non-parametric Mann-Whitney test. ${ }^{*} p<0.05$ compared with mice treated with clodronate liposomes. (D) Wild-type (WT) mice were killed at $24 \mathrm{~h}$ after sham or CLP surgery, and relative expression of IL-27R (WSX-1) mRNA was determined by quantitative PCR ( $n=6$ mice/group). Each symbol represents an individual subject and horizontal bars represent median values. (E) Cell-surface expression of IL-27R on AMs after CLP or sham surgery determined by flow cytometry. (F) AMs from WT and IL-27R ${ }^{-l-}$ mice were infected with $P$ aeruginosa (multiplicity of infection, 10$)$ in the presence or absence of recombinant IL-27 $(50 \mathrm{ng} / \mathrm{mL})$ and anti-IL-10 neutralising antibodies $(2 \mu \mathrm{g} /$ $\mathrm{mL}$ ). Extracellular bacteria were then removed by washing with tobramycin. Cells were lysed and live intracellular bacteria were determined by culture for evaluation of bacterial uptake $(\mathrm{t}=0)$ and intracellular killing $(\mathrm{t}=2 \mathrm{~h})$. Data were expressed as mean $\pm S E M$ and analysed using MannWhitney test (D and E) or one-way analysis of variance (ANOVA) (F). ${ }^{*} \mathrm{p}<0.05$ when compared between groups denoted by horizontal lines. i.t., intratracheal; PA, $P$ aeruginosa. 

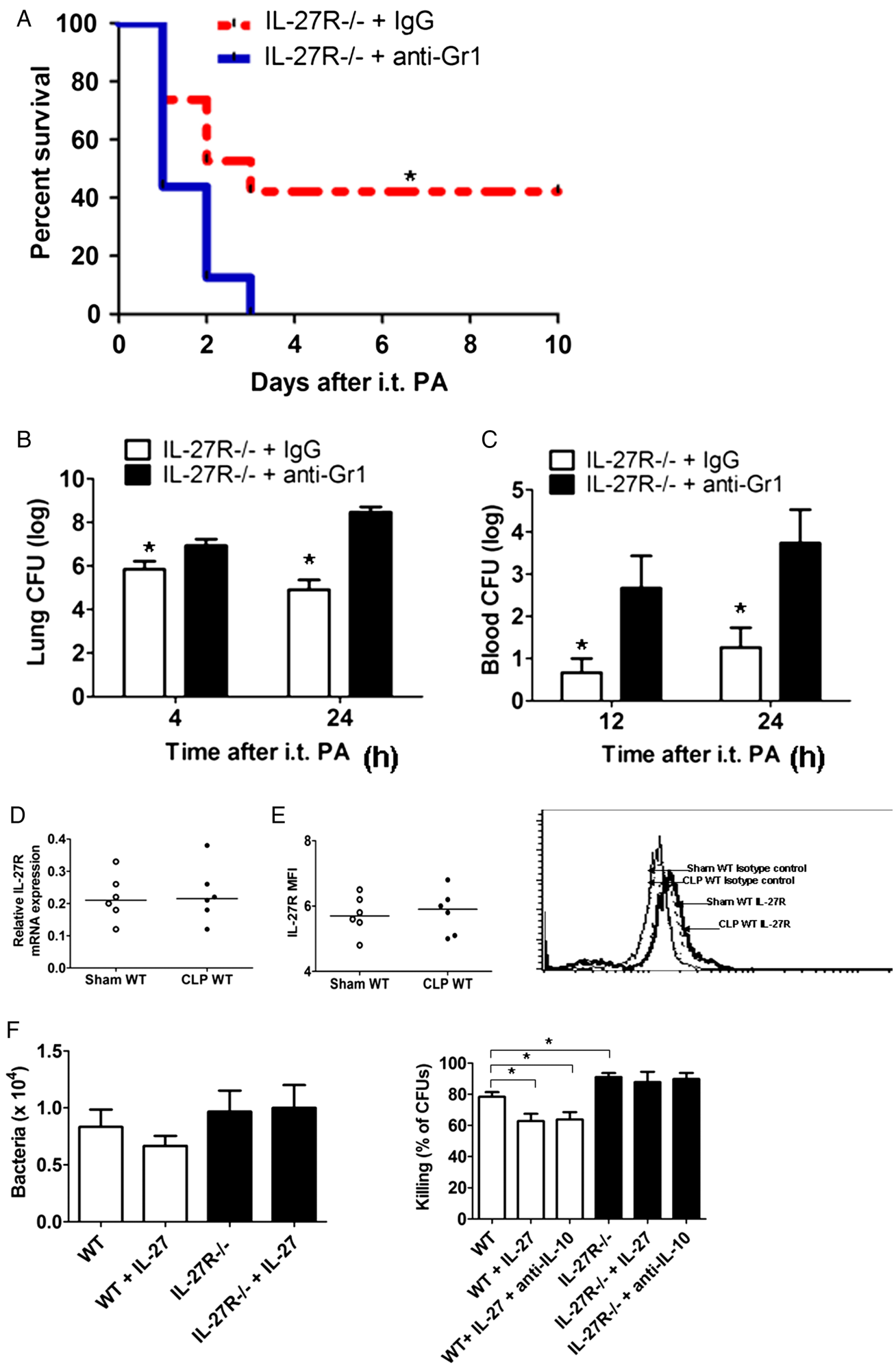

Figure 8 Interleukin (IL)-27 modulated immune responses of neutrophils. (A) Survival of septic IL-27R ${ }^{-1-}$ mice depleted of neutrophils upon secondary Pseudomonas aeruginosa infection. IL-27R ${ }^{-l-}$ mice underwent caecal ligation and puncture (CLP) surgery; $24 \mathrm{~h}$ later, mice were given anti-Gr-1 antibodies or IgG control followed by secondary $P$ aeruginosa challenge $24 \mathrm{~h}$ later ( $\mathrm{n}=16$ mice/group). Survival curves were analysed using the log-rank (Mantel-Cox) test. ${ }^{*} \mathrm{p}<0.05$ compared with mice treated with anti-Gr-1 antibodies. (B and C) Lung and blood colony-forming units (CFUs) in IL-27R ${ }^{-1-}$ mice depleted of neutrophils upon secondary $P$ aeruginosa infection ( $n=6$ mice/group); data were expressed as mean \pm SEM and analysed using the non-parametric MannWhitney test. * $p<0.05$ compared with mice treated with anti-Gr-1 antibodies. (D) Neutrophils were purified from the bone marrow of wild-type (WT) mice at $24 \mathrm{~h}$ after CLP surgery, and relative expression of IL-27R (WSX-1) mRNA was determined by quantitative PCR ( $\mathrm{n}=6$ mice/group). (E) Cell-surface expression of IL-27R on neutrophils after CLP or sham surgery determined by flow cytometry. (F) Neutrophils from WT and IL-27R ${ }^{-l-}$ mice were infected with $P$ aeruginosa (multiplicity of infection, 100$)$ in the presence or absence of recombinant IL-27 (50 ng/mL) and anti-IL-10 neutralising antibodies $(2 \mu \mathrm{g} / \mathrm{mL})$. Cells were washed with buffer containing tobramycin to remove extracellular bacteria and were lysed. Live intracellular bacteria were counted by culture of lysates for determination of bacterial uptake $(t=0)$ and intracellular killing $(t=1 \mathrm{~h})$. Data were expressed as mean $\pm S E M$ and analysed using Mann-Whitney test $(D$ and $E)$ or one-way analysis of variance (ANOVA) ( $\mathrm{F}$ ); ${ }^{*} \mathrm{p}<0.05$ when compared between groups denoted by horizontal lines. i.t., intratracheal; PA, $P$ aeruginosa. 
IL-27 treatment enhanced TNF- $\alpha$ and IL-1 $\beta$ production from stimulated neutrophils (see online supplementary figure S2A). However, when the expression of adhesion molecules Mac-1 and leukocyte function-associated antigen 1 (LFA-1) on neutrophils were assayed, we found that IL-27 could decrease surface expression of Mac-1 but not LFA-1 on neutrophils (see online supplementary figure S2B). In addition, anti-IL-10 antibodies had no effects on these functions mediated by IL-27 in neutrophils.

\section{DISCUSSION}

Sepsis ranks in the top 10 causes of death worldwide. ${ }^{11}$ Most sepsis patients do not die from an overwhelming proinflammatory immune response but in an immunosuppressive state, which leads to increased susceptibility to secondary (opportunistic) infections. 71123 The current study found that IL-27 regulated the increased susceptibility to secondary $P$ aeruginosa pneumonia in septic mice. Importantly, IL- $27 \mathrm{R}^{-/-}$mice were more resistant to secondary bacterial pneumonia in the septic setting, with strikingly lower lung and blood bacterial burdens and increased survival compared with WT mice. These findings demonstrate that IL-27 induced during sepsis sensitises hosts to secondary bacterial infections in the post-sepsis period.

Here we used a non-lethal CLP model before induction of pneumonia, and IL-27R $\mathrm{R}^{-/-}$and WT mice had survival rates $>90 \%$ following CLP. This was in contrast to previous findings that mice deficient for the EBI3 subunit of IL-27 were resistant to sepsis in a lethal CLP model. ${ }^{24}$ In light of substantially increased IL-27 expression levels in the lung after CLP, we were interested in investigating the role of IL-27 in sepsis-induced suppression of host defence in the lung. We reported that IL-27R deficiency protected mice against secondary $P$ aeruginosa pneumonia during CLP-induced sepsis, and IL-27 expression promotes the development of secondary $P$ aeruginosa pneumonia. These results in animal studies are consistent with recent clinical findings that IL-27 was a novel candidate diagnostic biomarker for predicting bacterial infection in patients with sepsis. ${ }^{25} 26$ Considering that IL-27 was elevated in patients with sepsis, targeting IL-27 may provide a new therapeutic approach for preventing patients with sepsis from secondary bacterial infections.

To understand the protection against $P$ aeruginosa pneumonia afforded by IL-27R deficiency in septic mice, lung cytokine/chemokine production following $P$ aeruginosa challenge in septic WT and IL-27R ${ }^{-1-}$ mice was determined. Compared with WT counterparts, septic IL-27 $\mathrm{R}^{-/-}$mice displayed significantly higher levels of TNF- $\alpha$, IL-1 $\beta$, CXCL1, CXCL2, CXCL10, IL-6, IL-12 and IL-17 $4 \mathrm{~h}$ after secondary $P$ aeruginosa infection. This upregulated cytokine/chemokine profile was associated with increased early neutrophil recruitment in the lung in IL-27R $\mathrm{R}^{-/-}$ mice. Several studies have addressed the inhibitory effects of IL-27 on T helper 1 (Th1), Th2 and Th17 cell responses. ${ }^{12} 27$ Interestingly, post-septic IL-27R $\mathrm{R}^{-1-}$ mice had lower concentrations of IL-10 at $24 \mathrm{~h}$ after secondary $P$ aeruginosa infection. Therefore, the upregulation of early proinflammatory response and downregulation of anti-inflammatory IL-10 in septic IL-27R $\mathrm{R}^{-/-}$mice may contribute to improved bacterial clearance.

As described previously, ${ }^{19-22}$ AMs and neutrophils were essential for rapid clearance of invading bacteria in the lung. Interestingly, IL-27R was upregulated in AMs but not in neutrophils during sepsis and mediated the responsiveness of AMs and neutrophils to $P$ aeruginosa. AMs from septic IL-27R ${ }^{-1-}$ mice had higher bacterial uptake and killing capacities, enhanced cytokine/chemokine production, and increased expression of costimulatory molecules compared with those from WT mice. Recombinant IL-27 could inhibit antimicrobial activity and cytokine/chemokine in WT AMs. In neutrophils, IL-27R $\mathrm{R}^{-/-}$neutrophils from septic mice also had an increased killing ability compared with WT neutrophils. Lung recruitment of neutrophils from the circulation to penetrate the blood vessels after infection relies on adherence of neutrophils to endothelial cells, which is associated with two adhesion molecules Mac-1 and LFA-1 on neutrophils. ${ }^{28}$ Intriguingly, we found that IL-27R ${ }^{-1-}$ neutrophils had a significantly higher expression of Mac-1 compared with WT neutrophils after CLP, suggesting that IL-27 might suppress neutrophil adhesion through downregulating the expression of Mac-1. Although recombinant IL-27 could also significantly decrease bacterial killing ability and Mac-1 expression in neutrophils, it enhanced IL- $1 \beta$ and TNF- $\alpha$ production by neutrophils upon the stimulation of $P$ aeruginosa, indicating that these proinflammatory effects of IL-27 on neutrophils were different from immunosuppressive effects on AMs. It has been reported that IL-27-mediated IL-10 production contributes to an anti-inflammatory role of IL-27 in T cells, ${ }^{12}{ }^{29}$ and IL-10 is an important cytokine mediator of sepsis-induced immunosuppression. ${ }^{6}$ However, there was no significant difference in IL-10 produced in the supernatants by WT and IL-27R ${ }^{-/-} \mathrm{AMs}$ and neutrophils upon $P$ aeruginosa stimulation (data not shown), and anti-IL-10 blocking antibodies showed no apparent effects on IL-27-mediated effects on AMs or neutrophils, indicating that these immunoregulatory effects of IL-27 on AMs and neutrophils may not be dependent on IL-10. Taken together, these results confirm a central role for IL-27 in promoting infection during the early phases of host defence response in septic hosts by regulating the responses of AMs and neutrophils. However, other immune cells including CD4, CD8 and $\gamma \delta \mathrm{T}$ cells, and natural killer cells also express IL-27R. ${ }^{13} 2730$ We cannot exclude a possibility that IL-27 may also regulate the functions of these cell types in the late-phase response to secondary $P$ aeruginosa in septic hosts, which requires further studies.

Overall, here we established that IL-27 impairs lung host immune responses against secondary bacterial challenge in a sublethal sepsis model. Our findings enlarge the understanding of pathophysiology during sepsis and may have important therapeutic implications.

Contributors Conception hypothesis and design: JC, FX and YY; data acquisition and analysis: JC, FX, SL, ZS, DL, LZ, PL, HX, KZ and GR; manuscript preparation: JC and FX.

Funding This work was supported by National Natural Science Foundation of China grants 81370110 (to JC), Natural Science Foundation grants of Chongqing 2011 BB5139 (to JC), and National Key Clinical Specialties Construction Program of China to the Department of Laboratory Medicine of the First Affiliated Hospital of Chongqing Medical University (No. 2010305).

Competing interests None.

Patient consent Obtained.

Ethics approval Clinical Research Ethics Committee of The First Affiliated Hospital of Chongqing Medical University.

Provenance and peer review Not commissioned; externally peer reviewed.

\section{REFERENCES}

1 For sepsis, the drugs don't work. Lancet Infect Dis 2012;12:89.

2 Angus DC, Wax RS. Epidemiology of sepsis: an update. Crit Care Med 2001;29 (Suppl. 7):S109-16

3 Hotchkiss RS, Karl IE. The pathophysiology and treatment of sepsis. N Engl J Med 2003:348:138-50.

4 Hotchkiss RS, Coopersmith CM, McDunn JE, et al. The sepsis seesaw: tilting toward immunosuppression. Nat Med 2009;15:496-7. 
5 Brun-Buisson C, Doyon F, Carlet J, et al. Incidence, risk factors, and outcome of severe sepsis and septic shock in adults. A multicenter prospective study in intensive care units. French ICU Group for Severe Sepsis. JAMA 1995;274:968-74.

6 Steinhauser ML, Hogaboam CM, Kunkel SL, et al. IL-10 is a major mediator of sepsis-induced impairment in lung antibacterial host defense. J Immunol 1999;162:392-9.

7 Hotchkiss RS, Opal S. Immunotherapy for sepsis-a new approach against an ancient foe. N Engl J Med 2010;363:87-9.

8 Brahmamdam $\mathrm{P}$, Inoue $\mathrm{S}$, Unsinger J, et al. Delayed administration of anti-PD-1 antibody reverses immune dysfunction and improves survival during sepsis. I Leukoc Biol 2010;88:233-40.

9 Inoue S, Bo L, Bian J, et al. Dose-dependent effect of anti-CTLA-4 on survival in sepsis. Shock 2011;36:38-44.

10 Kobayashi Y, Iwata A, Suzuki K, et al. B and T lymphocyte attenuator inhibits LPS-induced endotoxic shock by suppressing toll-like receptor 4 signaling in innate immune cells. Proc Natl Acad Sci U S A 2013;110:5121-6.

11 Leentjens J, Kox M, van der Hoeven JG, et al. Immunotherapy for the adjunctive treatment of sepsis: from immunosuppression to immunostimulation. Time for a paradigm change? Am J Respir Crit Care Med 2013;187:1287-93.

12 Hunter CA, Kastelein R. Interleukin-27: balancing protective and pathological immunity. Immunity 2012;37:960-9.

13 Pflanz S, Hibbert L, Mattson J, et al. WSX-1 and glycoprotein 130 constitute a signal-transducing receptor for IL-27. J Immunol 2004;172:2225-31.

14 Murugaiyan G, Beynon V, Pires Da Cunha A, et al. IFN- $\gamma$ limits Th9-mediated autoimmune inflammation through dendritic cell modulation of IL-27. J Immunol 2012;189:5277-83

15 Siebler J, Wirtz S, Frenzel C, et al. Cutting edge: a key pathogenic role of IL-27 in T cell-mediated hepatitis. J Immunol 2008;180:30-3.

16 Iyer SS, Ghaffari AA, Cheng G. Lipopolysaccharide-mediated IL-10 transcriptional regulation requires sequential induction of type I IFNs and IL-27 in macrophages. J Immunol 2010;185:6599-607.

17 Rinchai D, Khaenam P, Kewcharoenwong C, et al. Production of interleukin-27 by human neutrophils regulates their function during bacterial infection. Eur I Immunol. 2012:42:3280-90.
18 Levy MM, Fink MP, Marshall JC, et al. 2001 SCCM/ESICM/ACCP/ATS/SIS International Sepsis Definitions Conference. Crit Care Med 2003;31: $1250-6$.

19 Deng JC, Cheng G, Newstead MW, et al. Sepsis-induced suppression of lung innate immunity is mediated by IRAK-M. J Clin Invest 2006:116:2532-42.

20 Pène $F$, Grimaldi $D$, Zuber B, et al. Toll-like receptor 2 deficiency increases resistance to Pseudomonas aeruginosa pneumonia in the setting of sepsis-induced immune dysfunction. J Infect Dis 2012;206:932-42.

21 Manicone AM, Birkland TP, Lin M, et al. Epilysin (MMP-28) restrains early macrophage recruitment in Pseudomonas aeruginosa pneumonia. J Immunol 2009;182:3866-76.

22 Hubbard LL, Ballinger MN, Thomas PE, et al. A role for IL-1 receptor-associated kinase-M in prostaglandin E2-induced immunosuppression post-bone marrow transplantation. J Immunol 2010;184:6299-308.

23 Dalli J, Norling LV, Montero-Melendez T, et al. Microparticle alpha-2-macroglobulin enhances pro-resolving responses and promotes survival in sepsis. EMBO Mol Med 2014;6:27-42.

24 Wirtz S, Tubbe I, Galle PR, et al. Protection from lethal septic peritonitis by neutralizing the biological function of interleukin 27. J Exp Med 2006;203:1875-81.

25 Wong HR, Cvijanovich NZ, Hall M, et al. Interleukin-27 is a novel candidate diagnostic biomarker for bacterial infection in critically ill children. Crit Care 2012;16: R213.

26 Scicluna BP, van der Poll T. Interleukin-27: a potential new sepsis biomarker exposed through genome-wide transcriptional profiling. Crit Care 2012:16:188.

27 Passos ST, Silver JS, O'Hara AC, et al. IL-6 promotes NK cell production of IL-17 during toxoplasmosis. J Immunol 2010;184:1776-83.

28 Ding ZM, Babensee JE, Simon SI, et al. Relative contribution of LFA-1 and Mac-1 to neutrophil adhesion and migration. J Immunol 1999;163:5029-38.

29 Awasthi A, Carrier Y, Peron JP, et al. A dominant function for interleukin 27 in generating interleukin 10-producing anti-inflammatory T cells. Nat Immunol 2007:8:1380-9.

30 Morandi F, Prigione I, Airoldi I. Human TCR $\gamma \delta+\mathrm{T}$ cells represent a novel target for IL-27 activity. Eur J Immunol 2012:42:1547-52. 


\section{Correction}

Cao J, Xu F, Lin S, et al. IL-27 controls sepsis-induced impairment of lung antibacterial host defence. Thorax 2014;69:926-37. doi: 10.1136/thoraxjnl-2014-205777.

'The Affiliated Hospital of Chongqing Medical University' in the first 2 affiliations and the corresponding author's address should be corrected to 'The First Affiliated Hospital of Chongqing Medical University'.

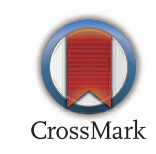

Thorax 2015;70:243. doi:10.1136/thoraxjnl-2014-205777corr1 